\title{
Expression, Purification, and Antiserum Production of the Truncated UL31 Protein of Herpes Simplex Virus 1
}

\author{
Xingmei Zou ', Zuo Xu ${ }^{1}$, Yuanfang Wang ', Xiaowen Ou ${ }^{1}$, Yiwen Li ${ }^{1}$, Delong Liu ${ }^{2}$, Weidong Gan ${ }^{2}$, \\ Manjiao Lu ${ }^{2}$, Qiusan Chen ${ }^{3}$, Hao Peng ${ }^{2}$, Gengde Hong ${ }^{3}$, Jinyu Lin ${ }^{3}$, Meili Li 1,4, , Mingsheng Cai ${ }^{1, *}$ \\ ${ }^{1}$ Department of Pathogenic Biology and Immunology, Sino-French Hoffmann Institute, School of Basic Medical \\ Science, Guangzhou Medical University, Xinzao Town, Panyu, Guangzhou 511436, Guangdong, China \\ ${ }^{2}$ GMU-GIBH Joint School of Life Sciences, Guangzhou Medical University, Xinzao Town, Panyu, Guangzhou 511436, \\ Guangdong, China \\ 3 The Third Clinical School of Guangzhou Medical University, No. 63 Duobao Road, Liwan District, Guangzhou \\ 510150, Guangdong, China \\ ${ }^{4}$ Guangdong Provincial Key Laboratory of Allergy and Clinical Immunology, Second Affiliated Hospital of Guangzhou \\ Medical University, No.250 Changgang Dong Road, Haizhu District, Guangzhou 510260, Guangdong, China
}

* Corresponding author: Meili Li and Mingsheng Cai. Department of Pathogenic Biology and Immunology, Guangzhou Medical University, Xinzao town, Panyu, Guangzhou 511436, Guangdong, P.R. China. Tel/Fax: +11-862037103216, E-mail: meili_2011@hotmail.com, caimingsheng@163.com

Background: The UL31 protein of herpes simplex virus 1 (HSV-1) plays an important role in the HSV-1 replication, however, its pinpoint functions in the life cycle of the virus have yet to be adequately elucidated.

Objectives: An antiserum specific for detecting HSV-1 UL31 was prepared as the foundation for future research on the role of UL31 in the course of HSV-1 infection.

Materials and Methods: Recombinant protein of UL31 was expressed in Escherichia coli, which was then purified and employed to raise the level of antiserum in mice. Subsequently, western blot and immunofluorescence assay (IFA) were utilized to detect the specific antiserum.

Results: The recombinant UL31 protein consisting of N-terminal 27 aa of UL31 was fused to EYFP and His-tag. It was expressed, purified, and applied to the preparation of the antiserum. Western blot analysis and IFA demonstrated that this antiserum could detect both the recombinant UL31 and the native UL31.

Conclusions: Our results manifest that this antiserum could be conducive to further investigations concerning the roles of UL31 in the HSV-1 infection.

Keywords: Escherichia coli; Herpes simplex virus 1; Immune Sera; Recombinant Proteins

\section{Background}

It is well-known that HSV-1 (herpes simplex virus 1) is a common human virus that can cause a variety of diseases, including mucocutaneous lesions of the mouth, eyes, face, or genitalia, and, seldom, meningitis or encephalitis (1). The UL31 protein contains 306 amino acids (aa), encoded by the HSV-1 UL31 gene (24 ), and in the life cycle of the virus, UL31 is one of the important structural proteins that has certain characteristics, such as binding with HSV-1 UL34 to form the nuclear egress complex (2-4), altering localization, phosphorylation, and interaction of nuclear lamina components and breaking off the nuclear lamina (5-7), promoting primary envelopment and nuclear exit of nucleocapsids (8-10). Furthermore, UL31 might form a network to allow the anchorage of viral products for the synthesis and/or packaging of viral DNA into virions (11). Besides, UL31 is involved in both the optimal activation of NF- $\kappa \mathrm{B}$ and the expression of viral gene products (12). However, the precise role of UL31 in the HSV-1 infection remains to be further studied.

\section{Objectives}

In the current study, a recombinant protein composed of truncated N-terminal 1-27 amino acids (aa) of UL31 fused with His and EYFP tag was expressed in E. coli and purified by Ni-NTA affinity chromatography under denatured conditions. Following renaturation, the recombinant protein was employed to raise the level of the antiserum in the BALB/c mice. aa 1-27 of HSV-1 UL31 was chosen for preparing antiserum since this 
fragment is the nuclear localization signal (NLS) of UL31 (13) and its antiserum is important for elucidating the function of UL31 NLS in the course of the HSV-1 infection. Subsequently, the results of western blot analysis showed that both the truncated and the native UL31 proteins could be identified by this antiserum. Besides, immunofluorescence assay (IFA) disclosed the subcellular localization pattern of UL31 in cells infected with HSV-1.

\section{Materials and Methods}

\subsection{Plasmids Construct}

UL31 ORF (consisting of $918 \mathrm{bp}$ ) was amplified by PCR from the genomic DNA of HSV-1 F strain (accession No: KM222725) (14), using KOD-plusNeo (TOYOBO) with the following primers: UL31-F (5'-

CGAAGCTTCGGAATTCATGTATGACACCGAC
CCCCATC -3') and UL31-R (5'-
GCAAGCTTAGGATCCGTCGGCGGAGGAAACT CGTCGAA -3'). As performed in our previous efforts (4, 15-20), the purified fragment was treated with EcoRI and $\mathrm{BamHI}$ and ligated into the same treated pEYFP-N1 (Clontech) to get pUL31-EYFP. Then, the DNA fragment encoding N-terminal 27 aa of UL31 was amplified from $p U L 31$-EYFP using primers UL31-F and UL31 (1-27)-R (5'AAGGATCCAGAGAGGAGCGCGACCGCCGGC3'). The PCR product was inserted into PEYFP-N1 to produce pUL31 (1-27)-EYFP. Afterwards, the DNA product corresponding to the N-terminal 27 aa of UL31 fused to EYFP was digested with EcoRI and NotI, and was subcloned into the correspondingly digested pET$28 \mathrm{a}(+)$ (Novagen) to construct a recombinant prokaryotic expression plasmid - i.e., pET28a(+)UL31(1-27)-EYFP. For determining the presence of the target insert in the obtained recombinant plasmid pET28a (+)-UL31 (1-27)-EYFP by PCR, the primer EYFP-R GAAGATCTCGCTTGTACAGCTCGTCCATGCC G-3') was also applied.

\subsection{Expression and Purification of the Recombinant} Protein

E. coli BL21 (DE3) cells were transformed with the recombinant plasmid pET28a (+)-UL31 (1-27)-EYFP to express the recombinant protein. Next, the expression and the purification of the recombinant protein were manipulated as done before (20-23). To increase the production of the recombinant protein, the condition of the culture was optimized for expression by varying the temperatures, the concentrations of IPTG, and the duration of induction. Protein expression was assessed by SDS-PAGE. Soluble and insoluble fractions were then analyzed in parallel by $12 \%$ SDS-PAGE. The recombinant protein was purified on $\mathrm{Ni}^{2+}$-NTA affinity resin following the modified version of the manufacturer's instructions. The purified recombinant protein was stored at $4{ }^{\circ} \mathrm{C}$ for use within 1 week or at $70{ }^{\circ} \mathrm{C}$ for future use.

\subsection{The Preparation of the Antiserum}

The antiserum preparation was carried out as represented previously, except that the recombinant protein was employed in this research (21-23). The antiserum was harvested from the eyeball of the BALB/c mice and stored at $-80^{\circ} \mathrm{C}$ until further use.

\subsection{Western Blot Analysis}

Western blot analysis was manipulated as previously reported and we used the antiserum against the recombinant protein $(20,22,24)$.

3.5. IFA

To see the subcellular localization of UL31 in cells infected by HSV-1, we infected them with HSV-1 (F) strain with an MOI of 1 at $0 \mathrm{~h}, 12 \mathrm{~h}$, and $24 \mathrm{~h}$ post infection (hpi). Vero cells were subjected to IFA by formaldehyde-based fixation approach, by using the antiserum against the recombinant protein, as mentioned in our previous reports $(20,22)$. The cell nuclei were counterstained by Hoechst.

\section{Results}

4.1. The Plasmid Expressing the Truncated UL31 Recombinant Protein was constructed.

In an attempt to produce the specific antibody against HSV-1 UL31 the plasmids pUL31-EYFP and pUL31 (1-27)-EYFP were constructed (data not shown). The DNA fragment encoding UL31 (1-27)-EYFP was digested with EcoRI and NotI then was subcloned from pUL31 (1-27)-EYFP into the corresponding digested pET-28a (+) (Novagen) to yield a recombinant prokaryotic expression plasmid pET28a (+)-UL31 (127)-EYFP (Fig. 1, lane 3). Afterwards, the recombinant plasmid (pET28a (+)-UL31 (1-27)-EYFP) was examined by PCR (Fig. 1, lane 1 and lane 2 ) and was subjected to the restriction analysis (Fig. 1, lane 4). The results demonstrate that the plasmid was successfully constructed. In addition, DNA sequencing results show that, in contrast to the HSV-1 F strain, there was no nucleotide mutation in the truncated UL31 protein (data not shown).

\subsection{Expression and Purification of the Recombinant Protein}

Here we used E. coli strain BL21 (DE3) to express the recombinant UL31 protein. The strain has the advantage of lacking the omp $\mathrm{T}$ with lon proteases while it harbors the T7 bacteriophage RNA polymerase gene, which makes it possible for the heterologous genes to specifically expressed by the T7 promoter $(25,26)$. After induction for $4 \mathrm{~h}$ at $37^{\circ} \mathrm{C}$ with $1.0 \mathrm{mmoL} . \mathrm{L}^{-1}$ IPTG, although BL21 (DE3) containing the recombinant plasmid did not express under non- 
induced condition (Fig. 2A, lane 1), E. coli BL21 (DE3) containing the recombinant plasmid expressed in abundance after induction (Fig. 2A, lane 2). An evident band of about $35 \mathrm{kDa}$, corresponding to the expected $\mathrm{M}_{\mathrm{r}}$ of truncated UL31 (1-27)-EYFP-His protein was observed (Fig. 2A, lane 2).

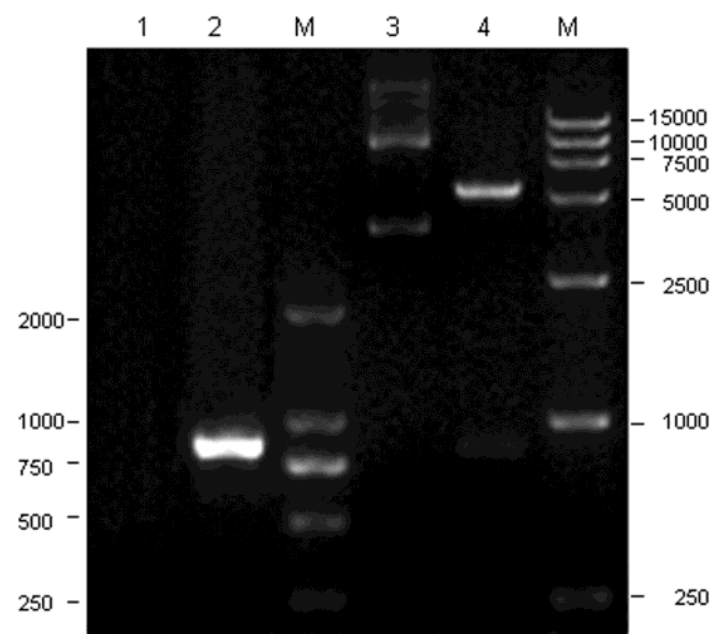

Figure 1. Cloning and verification of the recombinant plasmid pET28a-UL31 (1-27)-EYFP. The negative control (lane 1), PCR product with primers UL31F and EYFP-R (lane 2), the recombinant plasmid (lane 3), restriction enzyme digestion product of the recombinant plasmid with EcoRI and NotI (lane 4), DNA size markers (lanes M).

Several expression parameters, such as induction IPTG concentrations (Fig. 2A, lane 1-4) and induction time (Fig. 2A, lane 5-8) were manipulated to optimize the expression of the recombinant protein. The recombinant protein exhibited high expression after induction under all the conditions explored in this study. SDS-PAGE analysis indicated that the recombinant protein was largely insoluble in the form of inclusion bodies. Between cell debris pellet (Fig. 2B, lane 3) and the soluble fraction (Fig. 2B, lane 2), the induced recombinant protein was examined primarily in debris pellets (Fig. 2B, lane 3).

To establish an efficient approach to purify the recombinant UL31, an immobilized metal affinity chromatography on $\mathrm{Ni}^{2+}$-NTA resin column, which exhibits a high binding capacity allowing for a rapid and single-step purification, was applied to purify the recombinant protein (27). The successful purification of the purified recombinant protein is evident in SDSPAGE analysis, since only one clear band corresponding to $\mathrm{Mr}$ of about $35 \mathrm{kDa}$ was probed (Fig. 2C, lane $\mathbf{1}$ ). After purification, we can acquire about $240 \mathrm{mg}$ of recombinant protein per liter culture. Finally, the $\mathrm{BALB} / \mathrm{c}$ mice were injected with the purified protein to produce the specific antiserum against this recombinant protein.

\subsection{Characterization of the Antiserum against Recombinant Protein}

Three immunizations later, the antiserum was sampled from the eyeball of the BALB/c mice, subsequently western blot analysis was carried out to evaluate the specificity and the reactivity of the antiserum. The antiserum could recognize UL31 in the HSV-1 infected Vero cells (Fig. 3, lane 1). As expected, we could not locate a band from the mock-infected Vero cells (Fig. 3, lane 2). Furthermore, western blot analysis demonstrated that the antiserum could detect the fulllength UL31 tagged by EYFP protein in the lysates of pUL31-EYFP from the transfected HEK293T cells (Fig. 3, lane 3), whereas no band was observed in the lysates of the untransfected HEK293 T cells (Fig. 3, lane 4). These results suggest that the prepared antiserum has an adequate reactivity with the recombinant and also the native UL31.
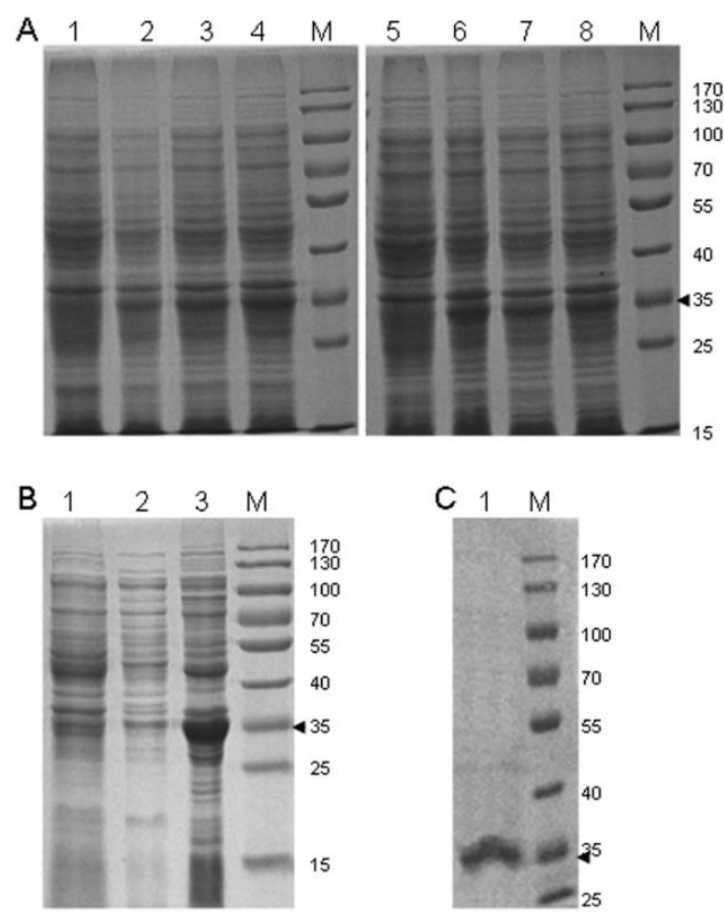

Figure 2. Expression analysis and expression optimization of the recombinant protein. (A) Optimization of the IPTG concentration and the induction duration. Lanes 1 to 4 , total protein from the recombinant plasmid transformed BL21 after induction with IPTG: 0 , $1.0,0.2,0.1 \mathrm{mM}$, at $37{ }^{\circ} \mathrm{C}$, respectively; lanes 5 to 8 , total protein from the recombinant plasmid transformed BL21 after induction with IPTG $(1.0 \mathrm{mM})$ for $0,4,8$ and $12 \mathrm{~h}$, at $37{ }^{\circ} \mathrm{C}$, respectively. (B) Expression analysis of the recombinant plasmid transformed BL21 non-induced (lane 1) and induced with IPTG separated in supernatant (lane 2) and pellet (lane 3). (C) Purification of the recombinant protein. Lane 1 , purified recombinant protein after dialysis. Lanes M, protein markers. Arrowheads indicate the position of the recombinant protein.

\subsection{Subcellular Localization of UL31 in HSV-1- Infected Cells}

IFA was performed at different times using the prepared antiserum to detect the subcellular localization pattern of UL31 in the HSV-1 infected Vero cells. We fixed and permeabilized the HSV-1-infected Vero cells at 0,12 
and 24 hpi. Next, the cells were blocked with BSA to remove nonspecific binding and allow for reaction with the prepared antiserum. As displayed in Figure 4, UL31 predominantly localized near the nuclear rim when cells were infected at 12 and $24 \mathrm{hpi}$. In contrast, we could not detect specific staining in the HSV-1-infected cells at 0 hpi. These results demonstrate that the prepared antiserum has adequate reactivity and specificity against the native UL31 proteins in the infected cells. Based the results, that UL31 is a nuclear rim-targeted protein.

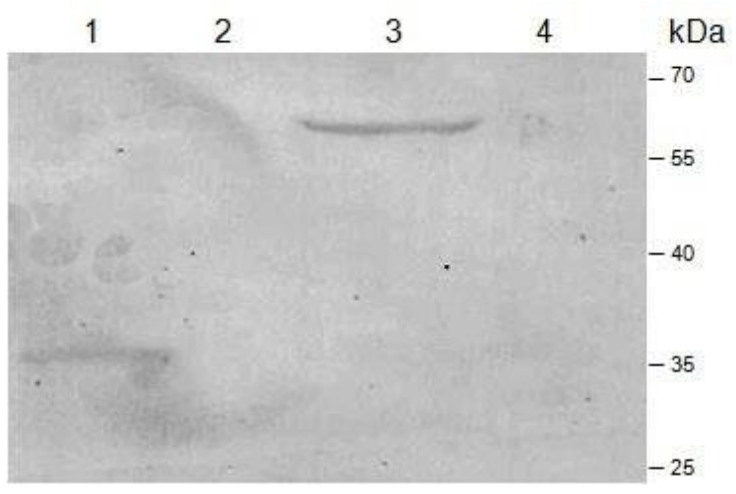

Figure 3. Characterization of the prepared antiserum against the recombinant protein by western blot analysis. Lysates of Vero cells infected with HSV-1 (lane 1) or mock infected (lane 2); lysates of HEK293T cells transfected with pUL31-EYFP (lane 3) or untransfected HEK293T cells (lane 4); the molecular mass of protein marker is labeled.

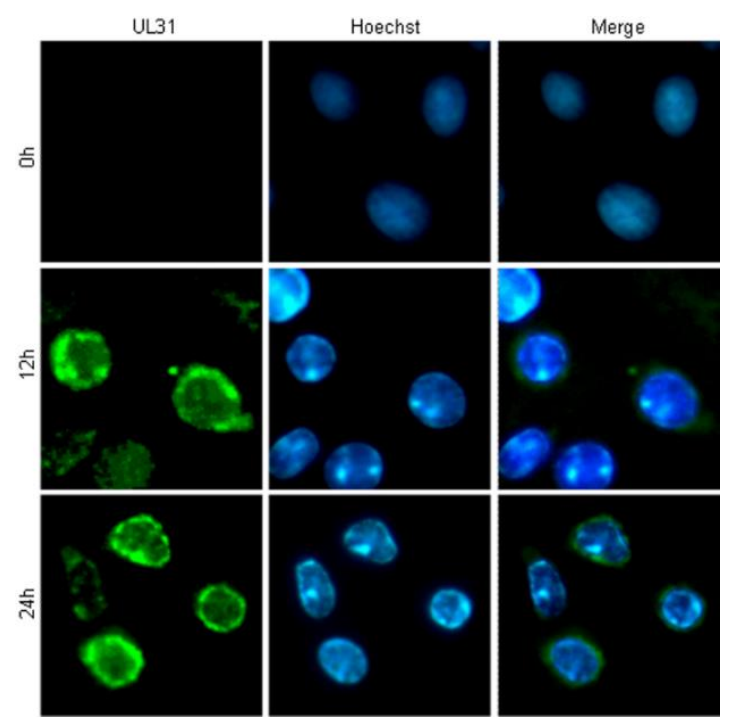

Figure 4. Subcellular localization of UL31 in HSV-1-infected VERO cells. Cells infected with HSV-1 F strain at an MOI of 1 . Then IFA was performed at the indicated times post infection with the antiserum against the native UL31 protein.

\section{Discussion}

Here we produced an antiserum against the truncated UL31 protein that can specifically recognize both the recombinant and the native UL31 proteins. As mentioned before, the precise role of UL31 in the HSV-
1 infection demands further investigation. Consequently, the prepared antiserum is a useful tool for in-depth study of the functions of UL31 or/and recombinant UL31.

It has been reported in that the intracellular localization of different viral proteins may reflect the various functions of the protein and the intracellular localization of viral proteins may also change at different times after infection (28). For example, it has been shown that in Vero cells infected with HSV-1, the localization of HSV1 UL3 changes from nuclear to small compact nucleosome during the viral replication cycle (29). Besides, HSV-1 UL31 and UL34 have separate but related functions in recruiting appropriate components to nucleocapsid budding sites at the inner nuclear membrane (30). One of the important properties of UL31 is its interaction with HSV-1 UL34, and this interaction is involved in several viral replication processes $(2,3)$, including (a) co-localization at the nuclear rim (31), (b) changing the localization, interaction, and phosphorylation of nuclear lamina components and disrupting the nuclear lamina (5-7), and (c) facilitating the primary envelopment and nuclear egress of their respective nucleocapsids (8-10). Therefore, it is expected that UL31 primarily displays nuclear rim localization in the HSV-1 infection cells (13, 32). Moreover, both the optimal activation of NF- $\kappa B$ and the expression of viral gene products involves UL31 (12), therefore it is not unexpected that UL31 is primarily a nuclear-associated protein.

\section{Conclusions}

In conclusion, we produced an antiserum against the truncated UL31 protein that could specifically recognize both the recombinant and the native UL31 proteins. Consequently, this antiserum could be considered a promising strategy to be used to further our understanding of the UL31 biological functions in the course of the HSV-1 infection.

\section{Acknowledgements}

We thank Dr. Chunfu Zheng (School of Basic Medical Sciences, Fujian Medical University) for the generous gift of HSV-1 virus.

\section{Funding / Support}

This work was supported by grants from the National Natural Science Foundation of China (81772179); the Natural Science Foundation of Guangdong Province (2018A0303130257 and 2015A030313473); the Regular University Distinguished Innovation Project from Education Department of Guangdong Province, China (2019LYM_0096); the Training Program for Outstanding Young Teachers in Universities of Guangdong Province (YQ2015132); the Medical Scientific Research Foundation of Guangdong Province, China (A2017055); the Science and 
Technology Plan Projects of Guangzhou City, China (201607010088); the Scientific Research Projects in Colleges and Universities of Guangzhou (1201610025 and 1201610024); High-Level Universities Academic Backbone Development Program of Guangzhou Medical University; and the Thousand Hundred Ten Projects of Guangzhou Medical University, Guangdong; the Undergraduate Laboratory Opening Project of Guangzhou Medical University (2018 and 2019); the National, Provincial and College Training Programs of Innovation and Entrepreneur-ship for Undergraduates in Guangzhou Medical University (pdjh2019b0406, 2018A113, 201810570030, 201810570057, 201710570007, 201710570010, 201710570065, 201710570070, 2018A113, 2017A035, 2017A070, 2017A075, 2016A003, 2016A066, XS201708 and XS201709)

\section{Financial Disclosure}

The authors declare that they have no competing interests.

\section{References}

1. Montgomery RI, Warner MS, Lum BJ, Spear PG. Herpes simplex virus-1 entry into cells mediated by a novel member of the TNF/NGF receptor family. Cell. 1996;87(3):427-436. doi: 10.1016/s0092-8674(00)81 363-x pmid: 8898196

2. Park R, Baines JD. Herpes simplex virus type 1 infection induces activation and recruitment of protein kinase $\mathrm{C}$ to the nuclear membrane and increased phosphorylation of lamin B. J Virol. 2006;80(1):494504. doi: 10.1128/JVI.80.1.494-504.2006 pmid: 16352573

3. Ye GJ, Roizman B. The essential protein encoded by the UL31 gene of herpes simplex virus 1 depends for its stability on the presence of UL34 protein. Proc Natl Acad Sci U S A. 2000;97(20):11002-11007. pmid: 11005871

4. Cai M, Zhao Z, Zhu J, Chen J, Wang B, Li Z, et al. Identification of Synonymous Codon Usage Bias in the Pseudorabies Virus UL31 Gene. Iran J Biotechnol. 2013;11(4):214-222. doi: 10.5812/ijb.13811

5. Reynolds AE, Liang L, Baines JD. Conformational changes in the nuclear lamina induced by herpes simplex virus type 1 require genes $\mathrm{U}(\mathrm{L}) 31$ and $\mathrm{U}(\mathrm{L}) 34$. JVirol. 2004;78(11):5564-5575. doi: 10.1128/JVI.78.1 1.5564-5575.2004 pmid: 15140953

6. Simpson-Holley M, Baines J, Roller R, Knipe DM. Herpes simplex virus $1 \mathrm{U}(\mathrm{L}) 31$ and $\mathrm{U}(\mathrm{L}) 34$ gene products promote the late maturation of viral replication compartments to the nuclear periphery. $J$ Virol. 2004;78(11):5591-5600. doi: 10.1128/JVI.78.1 1.5591-5600.2004 pmid: 15140956

7. Klupp BG, Granzow H, Fuchs W, Keil GM, Finke S, Mettenleiter TC. Vesicle formation from the nuclear membrane is induced by coexpression of two conserved herpesvirus proteins. Proc Natl Acad Sci U S A. 2007;104(17):7241-7246. doi: 10.1073/pnas.0701757 104 pmid: 17426144
8. Reynolds AE, Wills EG, Roller RJ, Ryckman BJ, Baines JD. Ultrastructural localization of the herpes simplex virus type 1 UL31, UL34, and US3 proteins suggests specific roles in primary envelopment and egress of nucleocapsids. J Virol. 2002;76(17):8939-8952. doi: 10.1128/jvi.76.17.8939-8952.2002 pmid: 12163613

9. Roller RJ, Bjerke SL, Haugo AC, Hanson S. Analysis of a charge cluster mutation of herpes simplex virus type 1 UL34 and its extragenic suppressor suggests a novel interaction between pUL34 and pUL31 that is necessary for membrane curvature around capsids. $J$ Virol. 2010;84(8):3921-3934. doi: 10.1128/JVI.0163809 pmid: 20106917

10. Mou F, Wills E, Baines JD. Phosphorylation of the $\mathrm{U}(\mathrm{L}) 31$ protein of herpes simplex virus 1 by the $\mathrm{U}(\mathrm{S}) 3$ encoded kinase regulates localization of the nuclear envelopment complex and egress of nucleocapsids. $J$ Virol. 2009;83(10):5181-5191. doi: 10.1128/JVI.000 90-09 pmid: 19279109

11. Chang YE, Van Sant C, Krug PW, Sears AE, Roizman B. The null mutant of the $\mathrm{U}(\mathrm{L}) 31$ gene of herpes simplex virus 1: construction and phenotype in infected cells. $J$ Virol. 1997;71(11):8307-8315. pmid: 9343183

12. Roberts KL, Baines JD. UL31 of herpes simplex virus 1 is necessary for optimal NF-kappaB activation and expression of viral gene products. $J$ Virol. 2011;85(10):4947-4953. doi: 10.1128/JVI.00068-11 pmid: 21389131

13. Cai M, Chen D, Zeng Z, Yang H, Jiang S, Li X, et al. Characterization of the nuclear import signal of herpes simplex virus 1 UL31. Arch Virol. 2016;161(9):23792385. doi: 10.1007/s00705-016-2910-z pmid: 27276 975

14. Wang K, Ni L, Wang S, Zheng C. Herpes simplex virus 1 protein kinase US3 hyperphosphorylates p65/RelA and dampens NF-kappaB activation. J Virol. 2014;88(14):7941-7951. doi: 10.1128/JVI.03394-13 pmid: 24807716

15. Li M, Jiang S, Mo C, Zeng Z, Li X, Chen C, et al. Identification of molecular determinants for the nuclear import of pseudorabies virus UL31. Arch Biochem Biophys. 2015;587:12-17. doi: 10.1016/j.abb.2015.0 9.024 pmid: 26450651

16. Li M, Jiang S, Wang J, Mo C, Zeng Z, Yang Y, et al. Characterization of the nuclear import and export signals of pseudorabies virus UL31. Arch Virol. 2015;160(10):2591-2594. doi: 10.1007/s00705-0152527-7 pmid: 26195191

17. Cai M, Jiang S, Zeng Z, Li X, Mo C, Yang Y, et al. Probing the nuclear import signal and nuclear transport molecular determinants of PRV ICP22. Cell Biosci. 2016;6(1):3. doi: 10.1186/s13578-016-0069-7 pmid: 26816613

18. Li M, Wang S, Cai M, Guo H, Zheng C. Characterization of molecular determinants for nucleocytoplasmic shuttling of PRV UL54. Virology. 2011;417(2):385-393. doi: 10.1016/j.virol.2011.06.0 04 pmid: 21777931

19. Li M, Wang S, Cai M, Zheng C. Identification of nuclear and nucleolar localization signals of pseudorabies virus (PRV) early protein UL54 reveals that its nuclear targeting is required for efficient production of PRV. $J$ Virol. 2011;85(19):10239-10251. doi: 10.1128/JVI.05 223-11 pmid: 21795331 
20. Cai M, Liao Z, Chen T, Wang P, Zou X, Wang Y, et al. Characterization of the subcellular localization of Epstein-Barr virus encoded proteins in live cells. Oncotarget. 2017;8(41):70006-70034. doi: 10.18632/o ncotarget. 19549 pmid: 29050259

21. Cai MS, Jiang S, Mo CC, Wang JL, Huang JL, Zeng ZC, et al. Preparation and identification of an antiserum against recombinant UL31 protein of pseudorabies virus. Acta Virol. 2015;59(3):295-299. doi: 10.4149/av_2015_03_295 pmid: 26435153

22. Li M, Cui $\bar{W}$, Mo $\bar{C}, \bar{W}$ ang J, Zhao Z, Cai M. Erratum to: Cloning, expression, purification, antiserum preparation and its characteristics of the truncated UL6 protein of herpes simplex virus 1. Mol Biol Rep. 2014;41(10):7031-7031. doi: 10.1007/s11033-0143696-2

23. Li ML, Li Z, Li WT, Wang BY, Ma CQ Chen JH, et al. Preparation and characterization of an antiserum against truncated UL54 protein of pseudorabies virus. Acta Virol. 2012;56(4):315-322. doi: 10.4149/av_201 2_04_315 pmid: 23237087

24. Cai M, Li M, Wang K, Wang S, Lu Q, Yan J, et al. The herpes simplex virus 1-encoded envelope glycoprotein $\mathrm{B}$ activates NF-kappaB through the Toll-like receptor 2 and MyD88/TRAF6-dependent signaling pathway. PLoS One. 2013;8(1):e54586. doi: 10.1371/journal.po ne.0054586 pmid: 23382920

25. Mierendorf RC, Morris BB, Hammer B, Novy RE. Expression and Purification of Recombinant Proteins Using the pET System. Methods Mol Med. 1998;13:257292. doi: 10.1385/0-89603-485-2:257 pmid: 21390849

26. Studier FW, Rosenberg AH, Dunn JJ, Dubendorff JW. Use of T7 RNA polymerase to direct expression of cloned genes. Methods Enzymol. 1990;185:60-89. pmid: 2199796

27. Arnau J, Lauritzen C, Petersen GE, Pedersen J. Current strategies for the use of affinity tags and tag removal for the purification of recombinant proteins. Protein Expr Purif. 2006;48(1):1-13. doi: 10.1016/j.pep.2005.12.0 02 pmid: 16427311

28. Feng ZP. An overview on predicting the subcellular location of a protein. In Silico Biol. 2002;2(3):291-303. pmid: 12542414

29. Lin F, Ren X, Guo H, Ding Q, Zheng AC. Expression, purification of the UL3 protein of herpes simplex virus type 1, and production of UL3 polyclonal antibody. $J$ Virol Methods. 2010;166(1-2):72-76. doi: 10.1016/j.j viromet.2010.02.022 pmid: 20188759

30. Wills E, Mou F, Baines JD. The U(L) 31 and U(L) 34 gene products of herpes simplex virus 1 are required for optimal localization of viral glycoproteins $\mathrm{D}$ and $\mathrm{M}$ to the inner nuclear membranes of infected cells. J Virol. 2009;83(10):4800-4809. doi: 10.1128/JVI.02431-08 pmid: 19279119

31. Reynolds AE, Ryckman BJ, Baines JD, Zhou Y, Liang L, Roller RJ. U(L)31 and $\mathrm{U}(\mathrm{L}) 34$ proteins of herpes simplex virus type 1 form a complex that accumulates at the nuclear rim and is required for envelopment of nucleocapsids. J Virol. 2001;75(18):8803-8817. doi: 10.1128/jvi.75.18.8803-8817.2001 pmid: 11507225

32. Cai M, Si J, Li X, Zeng Z, Li M. Characterization of the nuclear import mechanisms of HSV-1 UL31. Biol Chem. 2016;397(6):555-561. doi: 10.1515/hsz-2015-0299 pmid: 26854290 